\title{
Ethanol in Food affects behaviour and HPA axis activity during development in zebrafish larvae Subhead: Ethanol in Food affects Nerves during development in zebrafish larvae
}

wenxiao du ( $\square$ duwenxiao2007@163.com )

Yantai University

Xiaoli Chen

the fruit tree work station of penglai

Zhenjun Zhao

Yantai University

min shi

yantai university

fuhua bian

yantai university

\section{Research}

Keywords: Zebrafish, Food grade ethanol, HPA axis, Locomotor activity, neurotransmitter

Posted Date: January 23rd, 2020

DOI: https://doi.org/10.21203/rs.2.21719/v1

License: (c) (i) This work is licensed under a Creative Commons Attribution 4.0 International License.

Read Full License 


\section{Abstract}

Long-term alcohol intake from food can lead to numerous mental disorders in humans, and cause serious problems for governments and families worldwide. However, currently, it is unclear how alcohol affects the hypothalamic-pituitary-adrenal (HPA) axis. In the present study, using zebrafish larvae exposed to $1 \%$ ethanol, we made Zebrafish behavioural analysis, samples were collected for the enzymelinked immunosorbent assay (ELISA) and quantitative real-time polymerase chain reaction (qRT-PCR) experiments,and made statistical analyses at last. we found that ethanol decreases the locomotor activity of zebrafish larvae,showed a more intense reaction to external stimuli,increases the secretion of HPA axis hormones in zebrafish larvae,influences the secretion of neurotransmitters,alters key gene expression during neurotransmitter metabolism. Ethanol exposure reduced zebrafish locomotor activity, increased their HPA axis activity, and led to significant changes in the secretion of dopamine and serotonin. These findings provide us with a new understanding of the effects of ethanol on the HPA axis.

\section{Background}

Ethanol is a substance consume daily by humans that can cause considerable harm to human health (especially by minors)[1]. Indeed, the social and health effects of alcoholism are one of the most serious problems faced by governments and families every year[2]. Recent studies have found that long-term consumption of ethanol can lead to numerous physical[2], behavioural[3], and mental disorders[4].Alcohol consumption is a leading contributor to death from injuries, which itself is one of the main causes of death. For people under 21 years of age in the United States.Nearly 88,000 people die from alcohol-related causes annually, making alcohol the fourth leading preventable cause of death in the U.S[5].Furthermore, drinking during pregnancy can have a serious impact on foetal development, leading to foetal alcohol syndrome [6]. The main manifestations of this syndrome are skull deformity, cardiovascular dysplasia, and permanent nervous system damage[7]. Long-term drinking can also increase the risk of stroke, dementia, and hypertension; increase blood lipid concentration; and lead to a higher incidence of various cardiovascular and cerebrovascular diseases[8]. Because the liver and kidney are the main organs of alcohol metabolism, acute alcoholism can lead to alcoholic liver and kidney diseases[5]. The most common pathological changes of alcoholic liver are a decrease in the rough endoplasmic reticulum, swelling and deformation of the mitochondria, and the occurrence of liver cirrhosis[9]. Epidemiological studies have also shown that alcoholics are more likely to develop liver cancer. More recent studies have revealed that long-term drinking is associated with major mental disorders, such as depression[10].

Numerous studies on alcohol toxicity have been based on the zebrafish model[11]. The zebrafish (Danio rerio), as a vertebrate, has a genome with a high degree of homology with that of humans, this makes it a valuable model for developmental, neurological, and toxicological research[12]. Lockwood, for example, found that embryonic exposure to ethanol increases the susceptibility of larval zebrafish to chemically induced seizures[12]. Similarly matsui found that high concentrations of ethanol can lead to abnormal development of the visual system, while even low concentrations of ethanol can lead to behavioural 
disturbances[13]. Carva et al. found that the learning ability of adult and juvenile zebrafish treated with ethanol decreased significantly[14]. Accordingly, zebrafish are a good model for studying the toxicology of ethanol. While ethanol has been found to cause depressive phenotypes in behavioural experiments, the underlying mechanism of this effect remains unknown.

The hypothalamic-pituitary-adrenal (HPA) axis is a bodily system involving the hypothalamus, pituitary gland, and adrenal gland that is activated in response to environmental stress[15]. Specifically, the hypothalamus secretes adrenocorticotropin-releasing hormone (ACTH), which in turn acts on the pituitary gland to release adrenocorticotropin (CRH). $\mathrm{CRH}$ then acts on the adrenal gland to secrete glucocorticoids (GCs), which regulate HPA axis activity through the glucocorticoid receptors (gr or nr3c1) [16]. Cortisol is the main glucocorticoid in both humans and zebrafish, while corticosterone is the main glucocorticoid in rodents[17]. The HPA axis is an important aspect of the neuroendocrine system as it controls the stress response and regulates numerous physical activities, such as digestion, the immune system, mood and emotion, sexual behaviour, and energy storage and consumption[16]. Upstream in the HPA axis, numerous neurotransmitters are involved in regulating hypothalamic activity, which in turn regulates the activity of the entire HPA axis, including specific changes in mood[18]. Clinical studies have shown that a lot of patients with depression exhibit decreased GR expression and increased HPA axis activity[19]. At four days post-fertilization (dpf), zebrafish show a fully functional HPA axis, and the HPA axis of zebrafish has remarkably similar functions that of humans. Indeed, zebrafish have been successfully applied in studies on the HPA axis in humans. Thus, zebrafish offer a good model for studying the function of the HPA axis [20].

In this study, we exposed zebrafish larvae to $1 \%$ ethanol and examined their subsequent behaviour, hormones, neurotransmitters, and gene expression. The results showed that ethanol treatment reduced zebrafish activity and increased their response to environmental stimuli as well as their HPA axis activity. In addition, there were significant changes in the secretion of certain neurotransmitters. This study provides new understanding of the effects of ethanol on the HPA axis, and provide new evidence on whether or not alcohol-control.

\section{Methods And Materials}

Fish maintenance and ethanol treatment procedure

All experimental procedures were approved by the Yantai University Animal Care and Use Committee and were in accordance with governmental regulations of China.

Adult zebrafish of the $A B$ line were raised in a recirculating water system under a 14/10-h light/dark (L/D) cycle at $28^{\circ} \mathrm{C}$. They were fed three times per day. To produce embryos, male and female zebrafish were paired in the evening, and spawning occurred the next day within $1 \mathrm{~h}$ after the lights were switched on. The embryos were placed in 10-cm Petri dishes with egg water containing methylene blue (0.3 ppm), and raised in a light-controlled (14/10-h L/D) incubator at $28^{\circ} \mathrm{C}$. According to the data of our previous experiment, $1 \%$ ethanol significantly alters zebrafish larvae behaviour and gene expression, so at $24 \mathrm{~h}$ 
post-fertilization (hpf), the larvae were exposed to $1 \%$ ethanol, A control group consisting of sibling larvae raised in E3 solution were applied too. For each group, the ethanol solution was refreshed daily until the sampling stage. At 3, 4, 5, and $6 \mathrm{dpf}$, samples were collected for the enzyme-linked immunosorbent assay (ELISA) and quantitative real-time polymerase chain reaction (qRT-PCR) experiments.

Zebrafish behavioural analysis

Locomotor activity analysis was performed as described in a previous study, with some modifications. At $5 \mathrm{dpf}$, a single larva was placed in each the wells of a 48-well plate (24 control and 24 ethanol-exposed larvae), thereby allowing for simultaneous tracking of the larvae. The locomotor activities of the larvae were monitored for 30 min using an automated video-tracking system (Videotrack, ViewPoint LifeSciences), while their movement was recorded and analysed using Zebralab 3.10 software (ViewPoint Life Sciences). The 48-well plates were placed inside the Zebrabox Observation Chamber, wherein they were exposed to continuous infrared light or $10 \mathrm{~min}$ light $\mathrm{ON}$ and $10 \mathrm{~min}$ light OFF. Instruments were placed in the chamber to maintain a constant temperature of $28.5^{\circ} \mathrm{C}$. The Videotrack quantization parameters were set as in the previous study. The test was performed 3 times. Data were further analysed using Microsoft Excel.

\section{RNA extraction and qRT-PCR}

For the qRT-PCR analysis, total RNA was extracted using TRIzol Reagent (Invitrogen) according to the manufacturer's instructions. Following DNase treatment, RNA $(1 \mu \mathrm{g})$ was reverse-transcribed with oligo (dT) 18 primers and M-MLV reverse transcriptase. The qRT-PCR analysis was then carried out in the StepOne Real-Time PCR System (ABI) using SYBR® Premix Ex TaqTM (Takara Bio Inc., Dalian, Liaoning). At least three independent samples were examined in this analysis, each in triplicate. The mRNA level of the interested genes was calculated using 2- $\Delta$ CT method and presented as relative (-fold) values of the control group after normalization to the $\beta$-actin mRNA levels.

The target gene levels were normalized to $\beta$-actin, and for each sample triplicates were run. In each reaction with the target gene primers, $1 \mu$ l of undiluted cDNA was used, whereas the cDNA used for $\beta$ actin amplification was diluted 1:10. The relative expression of the target genes was calculated using the $2(-\Delta \Delta \mathrm{CT})$ method. The relative primers are listed in Table 1. 
Table 1

The relative primers of genes

\begin{tabular}{|llll|}
\hline Gene name & Forward primer & Reverse primer & Note \\
\hline crha & CAGCAGACTCTCACCGACAA & CAGAGCTCCAGACGGAGAGT & qRT-PCR \\
crhb & CTCGCCACTTTTTGACATGA & GCTGCTCTCGATGGCTCTAC & qRT-PCR \\
pomca & GCTCAGTGTTGGGAAAATGC & GGTAGACGGGGGTTTCATCT & qRT-PCR \\
pomcb & GTGCAGATCGGACCAAGAAT & GCAAACCCAAGCTCAGACTC & qRT-PCR \\
nr3c1 & GGCCAGTTTATGCTTTTCCA & TTGTGTGTGCCAGTCTTTCC & qRT-PCR \\
maoa & GAATCCTGTGGTCCTGGAAG & GAATGCGGTTTTGAGTTGGT & qRT-PCR \\
th & CAGCTCCACATCTTCCACAA & CGCATCCTCGATCAAACTCT & qRT-PCR \\
dbh & AAGAGCTCCATCATGGCATT & CTGCCTTCACTGTCACTCCA & qRT-PCR \\
B-actin & ATCACAGTTCCAGCCTATTTC & TGCCGTCTTCGATGGTCAG & qRT-PCR \\
\hline
\end{tabular}

Enzyme-linked immunosorbent assay (ELISA)

Samples from the ethanol and control groups in the 3, 4, 5 and $6 \mathrm{dpf}$ zebrafish were extracted. We analysed the HPA axis hormones and neurotransmitters using an ELISA kit obtained from Fanke Biotech (Shanghai, China) according to the manufacturer's protocol.

Statistical analyses

The data are presented below as the mean \pm SD. Statistical differences between two or more groups were determined using Student's t-test and one-way analysis of variance (ANOVA) followed by the StudentNewman-Keuls test, respectively. All experiments were repeated three times, and the representative results are described in Sect. 3. A p-value of $<0.05$ was considered to indicate statistical significance.

\section{Results}

Exposure to ethanol affects zebrafish behaviour

To investigate whether ethanol affects the autonomous behaviour of zebrafish, we selected the $120 \mathrm{hpf}$ control and ethanol-exposed zebrafish larvae for behavioural analysis. The results showed that under the same conditions, the swimming distance of zebrafish exposed to $1 \%$ ethanol was significantly shorter than that of the control fish (Fig. 1A), while the swimming speed per unit time was significantly faster (Fig. 1B). Furthermore, the average swimming time per unit time of the ethanol group was significantly lower than that of the control group (Fig. 1C). Taken together, these results suggest that exposure to ethanol decreases the locomotor activity of zebrafish larvae. 
To tests the exposed zebrafish's ability to respond to external stimuli, we observed their responsiveness in alternating stimulation of light and dark (30 minutes of light and 30 minutes of darkness). Compared to the control group, the ethanol group showed significantly reduced activity in the light condition (Fig. 1DE), but significantly greater swimming distance in the dark condition (Fig. 1D-E). These findings indicate that the ethanol-exposed zebrafish showed a more intense reaction to external stimuli.

Exposure to ethanol affects the secretion of HPA axis hormones

To investigate whether ethanol exposure affects the zebrafish HPA axis activity, we used the ELISA method to detect the secretion of related hormones. The results showed that the secretion of $\mathrm{CRH}$ was significantly elevated after exposure to ethanol at 3, 4, 5, and $6 \mathrm{dpf}$ (Fig. 2A), as was the secretion of ACTH (Fig. 2B) and the secretion of cortisol (Fig. 2C). Taken together, the results suggest that ethanol increases the secretion of HPA axis hormones in zebrafish larvae.

Exposure to ethanol affects the expression of HPA axis genes

Further to investigate how ethanol affects HPA axis activity, we performed a qRT-PCR analysis to investigate HPA axis gene expression. We found that the expression of crha showed no significant change at 3 and $4 \mathrm{dpf}$, but there was a significantly greater up-regulation at 5 and $6 \mathrm{dpf}$ in the ethanol group compared with the control group (Fig. 3A). Furthermore, the expression of crhb gene showed a statistically significant increase at 4,5, and $6 \mathrm{dpf}$ compared with the control group (Figure.3B); there was no significant difference at $3 \mathrm{dpf}$ (Fig. 3B). The expression of pomca and pomcb increased significantly with the development time. Specifically, the expression of pomca showed clear up-regulation at 4,5 , and $6 \mathrm{dpf}$, but there was not a statistically significant difference at $3 \mathrm{dpf}$ (Fig. 3C). The expression of pomcb did not show a significant differences at 3 and $4 \mathrm{dpf}$, but it did show significant up-regulation at 5 and 6 $\mathrm{dpf}$ in the ethanol group (Fig. 3D). The expression of nr3c1 increased with the development time, although there was a slight decrease in expression at $6 \mathrm{dpf}$ and the ethanol group did not show significant differences from the control group (Fig. 3E). Altogether, the HPA axis genes expression patterns of zebrafish larvae showed clear changes after exposure to ethanol.

Exposure to ethanol affects the secretion of neurotransmitters

Numerous monoamine neurotransmitters play an important role in the regulation of the HPA axis, particularly the regulation of individual behaviour and mood. To investigate whether ethanol exposure influences the secretion of neurotransmitters, particularly dopamine and serotonin, we used the ELISA method. The results showed that the secretion of dopamine showed a significantly greater increase at 3 , 5 , and $6 \mathrm{dpf}$ in the ethanol group than in the control group, although it decreased at $4 \mathrm{dpf}$ (Fig. 4A). Serotonin, on the other hand, showed significantly greater increases at all four time points in the ethanol group compared to the control group (Fig. 4B). These results indicate that exposure to ethanol influences the secretion of neurotransmitters.

Exposure to ethanol affects the expression of key genes in dopamine and serotonin metabolism 
Key genes act as rate-limiting enzymes in the synthesis and metabolism of certain neurotransmitters. We selected three key genes that served as rate-limiting enzymes for the above two neurotransmitters, and performed further qRT-PCR analysis. The results showed that compared with the control group, the expression of maoa significantly increased at 4, 5, and $6 \mathrm{dpf}$ in the ethanol group; however, there was no statistically significant difference at $3 \mathrm{dpf}$ (Fig. 5A). Furthermore, the expression of th was significantly greater in the ethanol group at 5 and $6 \mathrm{dpf}$, but showed no significant difference at 3 and $4 \mathrm{dpf}$ (Fig. 5B). Finally, the expression of dbh was significantly down-regulated at $5 \mathrm{dpf}$ in the ethanol treated group compared with the control group (Fig. 5C). Thus, the exposure to ethanol alters key gene expression during neurotransmitter metabolism.

\section{Discussion}

Zebrafish are a good model organism for neuroendocrine and behavioural research[20](Steenbergen et al.,2011). There are numerous established behavioural and physiological tests and methods of generating genetic mutations in zebrafish, and plenty of research using zebrafish to study ethanol toxicology [21]. In this study, we explored the effects of ethanol using behavioural experiments, hormone measurements, and gene expression, and found that ethanol led to decreased zebrafish locomotor activity, increased HPA axis activity, and neurotransmitter metabolism disorders.

Exposure to $1 \%$ ethanol led to changes in the swimming behaviour of zebrafish, including decreased swimming distance and increased speed. Furthermore, the light-dark experiment showed that that ethanol-exposed zebrafish were significantly more active in the dark condition. In the natural environment, darkness is a dangerous environment for animals [22]. This suggests that zebrafish exposed to ethanol showed a more anxious phenotype in response to danger. Previous studies have also found that after treated with ethanol, zebrafish adults and juveniles show anxiety-like behaviour, and this anxiety-like behaviour could be decreased using the antidepressant drug diazepam [23]. These results suggest that zebrafish juveniles exposed to ethanol exhibit a depression-like phenotype.

As noted above, the HPA axis is the main system by which animal response to changes in their external environment and stress, and the HPA axis of zebrafish and humans are exceedingly similar [24]. Past studies have shown that the shallow water test leads to a clear increase in cortisol secretion and HPA activity [25]. In the HPA axis, gr serves as a central molecule that shows decreased expression in depressed patients[26]. In zebrafish, deletion of the gr gene can also lead to mood disoder[27]. Based on these past findings, we examined the expression of key genes and secretion of hormones related to the HPA axis, and found that the expression of crh and pomca was significantly up-regulated at 5 and $6 \mathrm{dpf}$. Similarly, the secretion of hormones encoded by these two genes also increased significantly. Finally, ethanol exposure led to an increase in cortisol secretion. These data together indicate that ethanol leads to a significant increase in HPA axis activity.

Notably, however, while there was a decrease in the expression of the gr gene, this decrease was not statistically significant. This might be because the exposure time was insufficient to cause down- 
regulation of gr. In addition, we found no difference in the HPA axis activity at 3 and $4 \mathrm{dpf}$, possibly because the zebrafish larvae did not form a fully functional HPA axis before $4 \mathrm{dpf}[28]$. In addition, numerous compounds during pregnancy can lead to epigenetic changes in gr[29].which, along with development, have an impact on adolescents' personality and mood [30]. Whether embryo or maternal alcohol exposure can lead to differences in epigenetic regulation of zebrafish gr and mood changes in later developmental stages remains to be investigated in future studies.

The HPA axis is a neuroendocrine regulation system linked to the secretion of neurotransmitters associated with the development of mental illness[31]particularly dopamine[32] and serotonin[33]. We found significant increases in the secretion of both neurotransmitters after exposure to ethanol, along with increased expression of the maoa and th genes, and decreased expression of the dbh gene. The maoa [34], th [35] and dbh [36] genes are a rate-limiting enzymes of dopamine synthesis, serotonin synthesis, and dopamine metabolism, respectively. Thus, our results suggest that ethanol exposure increases the synthesis of dopamine and serotonin, while also reducing the metabolism of dopamine. Previous studies have also found that in the hypothalamus, dopaminergic and serotonergic neurons can project to ACTH neurons to regulate the HPA axis [37]; however, the detailed mechanism of this projection remains unclear. Whether ethanol exposure leads to changes in neurotransmitters than in turn produce behavioural changes, or whether ethanol exposure directly affects behavioural abnormalities caused by HPA axis activity, is a matter of further research.

Alcohol consumption is a major risk factor for road traffic crashes worldwide,and lead contributor to death from injuries. Drunk driving result significant economic costs, social costs (such as law enforcement,imprisonment, compensation payments, unemployment, disability in surance, and loss of productivity from injury or premature death).A lot of governments are adopting multipronged strategies to curb these costs, such as launched public service announcements, higher penalties for offenders, greater law enforcement presence on the roads, and so on[5]. Result of this research provide new evidence on development of these strategies for reducing drunk-driving fatal accidents. It has significant potential to stimulate meaningful in solution of various social problems.

\section{Conclusion}

Ethanol in food exposure reduced zebrafish locomotor activity, increased their HPA axis activity, and led to significant changes in the secretion of dopamine and serotonin. These results provide a new understanding of the effects of ethanol on the HPA axis.

\section{Declarations}

Funding

This work was supported by key research and development plan in Shandong Province(2019GSFI08220) and Natural Science Foundation Program of china (31971546). 
Conflicts of interest

The authors have no competing interests to declare.

Acknowledgements

We thank the support from yantai university biotechnology platform.

Author Disclosures

Ethics Approval and consent to participate

The adoption of zebrafish and all experimental procedures were approved by the Yantai University Animal Care and Use Committee and were in accordance with governmental regulations of China. Consent for Publication

All presentations of case reports have consent to publish.

Availability of supporting data.

"Availability of Data and Materials" section in this manuscript detailing where the data supporting their findings can be found.

Authors' contributions.

Wenxiaodu did this experiment and wrote some parts of the article.Other authors proofread and wrote part of the article.

All authors have read and understood Journal of Neuroinflammation's policies, and we believe that neither the manuscript nor the study violates any of these.All authors agree that this article can be published in the Journal of Neuroinflammation. This manuscript has not been published or presented elsewhere in part or in entirety and is not under consideration by another journal.

\section{References}

[1] Sánchez-Marín, L., Ladron, de, Guevara-Miranda, D., Carmen, Manas-Padilla, M., Alen, F., MorenoFernandez, D.R., Caridad Díaz-Navarro, Palacio Jose P, García-Fernandez M, Pedraza C, J. Pavon F, Rodríguez de Fonseca F, J. Santín L, Serrano A, Castilla-Ortega E., 2018. Systemic blockade of Ipa 1/3, lysophosphatidic acid receptors by ki16425 modulates the effects of ethanol on the brain and behavior. Neuropharmacology. 133, 189-201.

[2] Asatryan, L., Ostrovskaya, O., Lieu, D., Davies, D. L., 2017. Ethanol differentially modulates p2x4 and p2x7 receptor activity and function in bv2 microglial cells. Neuropharmacology. 128, 11-21. 
[3] Morais-Silva, G., Alves, G. C., Marin, M. T., 2016. N-acetylcysteine treatment blocks the development of ethanol-induced behavioural sensitization and related $\Delta$ fosb alterations. Neuropharmacology. $110,135-$ 142.

[4] Wang, X., Zhang, K., Yang, F., Ren, Z., Xu, M., Frank, J.A., Ke, Z.J, Luo, J., 2018. Minocycline protects developing brain against ethanol-induced damage. Neuropharmacology. 129, 84-99.

[5] Niederdeppe, J., Avery, R., Miller, E.N. 2017. Alcohol-control public service announcements (psas) and drunk-driving fatal accidents in the united states, 1996-2010.Prev Med. 99, 320-325.

[6] Khoury, J.E., Jamieson, B., Milligan, K., 2018. Risk for Childhood Internalizing and Externalizing Behavior Problems in the Context of Prenatal Alcohol Exposure: A Meta-Analysis and Comprehensive Examination of Moderators. Alcohol Clin Exp Res. 4, 1358-1377.

[7] Tielbeek, J.J, Vink J.M, Polderman T.J.C., Popma A, Posthuma D, Verweij K.J.H., 2018. Genetic correlation of antisocial behaviour with alcohol, nicotine, and cannabis use. Drug Alcohol Depen. 187, 296-299.

[8] Cheng, C.A., Lee, J.T., Lin, H.C., Lin, H.C., Chung, C.H., Lin, F.H., Tsao, C.H., Wu, Y.F., Chien, W.C., Chiu, $\mathrm{H}, \mathrm{W} ., 2017$. Pregnancy increases stroke risk up to 1 year postpartum and reduces long-term risk.Qjm-mon J Assoc Phys. 11(6), 355-360.

[9] Dreval, K., De Conti, A., Furuya, S., Beland, F.A., Rusyn, I., Pogribny, I.P. 2017. Mir-1247 blocks sox9mediated regeneration in alcohol- and fibrosis-associated acute kidney injury in mice. Toxicology. 384, 4049.

[10]Arora, P.G., Collins, T.A., Dart, E.H., Hernández, S., Fetterman, H., Doll, B., 2019. Multi-tiered systems of support for school-based mental health: a systematic review of depression interventions. Sch Ment Health. 2, 1-25.

[11]Getachew, B., Hudson, T., Heinbockel, T., Csoka, A.B., Tizabi, Y. 2018. Protective effects of donepezil against alcohol-induced toxicity in cell culture: role of caspase-3. Neurotox Res. 34,757-762.

[12]Desmidt, A. A., Zou, B., Grati, M., Yan, D., Mittal, R., Yao, Q., Richmond, M.T. , Denyer, S., Liu, X.Z., Lu Z., 2019. Zebrafish model for non-syndromic x-linked sensorineural deafness, dfnx1. Anat Rec. 1-12.

[13]Lockwood, B., Bjerke, S., Kobayashi, K., Guo, S., 2004. Acute effects of alcohol on larval zebrafish: a genetic system for large-scale screening. Pharmacol Biochem Be. 77(3), 647-654.

[14]Carvan, M.J.3rd., Loucks, E., Weber, D.N., Williams, F.E., 2004.Ethanol effects on the developing zebrafish: neurobehavior and skeletal morphogenesis. Neurotoxicol Teratol. 26, 757-768.

[15]Salgado-Freiría, R., López-Doval, S., Lafuente, A., 2018. Perfluorooctane sulfonate (pfos) can alter the hypothalamic-pituitary-adrenal (hpa) axis activity by modifying crf1 and glucocorticoid receptors. Toxicol 
Lett. 295, 1-9.

[16] Yue, N. , Renjie, C. , Yongjie, X. , Jing, C. , Zhekang, Y. , \& Zhijing, L. , et al. (2018). Fine particulate matter constituents and stress hormones in the hypothalamus-pituitary-adrenal axis. Environment International, 119, 186-192.

[18] Feder, A., Nestler, E.J., Charney, D.S., 2009.Psychobiology and molecular genetics of resilience. Nat Rev Neurosci 10, 446-457.

[19\} Esmaeili, M.H., Bahari, B., Salari, A.A., 2018.ATP-sensitive potassium-channel inhibitor glibenclamide attenuates HPA axis hyperactivity, depression- and anxiety-related symptoms in a rat model of Alzheimer's diseaseBrain Research Bulletin. 137, 265-276.

[20] Steenbergen, P.J., Richardson, M.K., Champagne, D.L. 2011.The use of the zebrafish model in stress research. Prog Neuro-Psychoph 35,1432-1451.

[21] Peng, J., Wagle, M., Mueller, T., Mathur, P., Lockwood, B.L., Bretaud, S., Guo, S., 2009.Ethanolmodulated camouflage response screen in zebrafish uncovers a novel role for CAMP and extracellular signal-regulated kinase signaling in behavioral sensitivity to ethanol. J Neurosci. 29, 8408-8418.

[22] Mathur, P., Guo, S., 2011. Differences of acute versus chronic ethanol exposure on anxiety-like behavioral responses in zebrafish. Behav Brain Res. 219(2), 230-239.

[23]Ramlan, N.F., Sata, N.S.A.M., Hassan, S.N., Bakar, N.A., Ahmad, S., Zulkifli, S.Z., Abdullah, C.A.C., Ibrahim, W.N.W., 2017.Time dependent effect of chronic embryonic exposure to ethanol on zebrafish: morphology, biochemical and anxiety alterations.Behav Brain Res. 332, 40-49.

[24]Liu, C., Zhang, X., Deng, J., Hecker, M., Al-Khedhairy, A., Giesy, J.P., Zhou, B., 2011. Effects of prochloraz or propylthiouracil on the cross-talk between the HPG, HPA, and HPT axes in zebrafish. Environ Sci Technol. 45, 769-775.

[25]Ramsay, J.M., Feist, G.W., Varga, Z.M., Westerfield, M., Kent, M.L., Schreck, C.B., 2009.Whole-body cortisol response of zebrafish to acute net handling stress. Aquaculture. 297, 157-162.

[26]Werne, B.C.V., Severi, M.C.M., Tofoli, Sandra, Márcia, de, C., Juruena, Mário, F., 2014. Early life stress in depressive patients: hpa axis response to gr and mr agonist. Front Psychiatry. 5,1-12.

[27]Ziv, L., Muto, A., Schoonheim, P.J., Meijsing, S.H., Strasser, D., Ingraham, H.A., Schaaf, M.J., Yamamoto, K.R., Baier, H., 2013. An affective disorder in zebrafish with mutation of the glucocorticoid receptor. Mol Psychiatr. 18, 681-691.

[28] Zhao, J., Xu, T., Yin, D.Q., 2014. Locomotor activity changes on zebrafish larvae with different 2,2',4,4'tetrabromodiphenyl ether (pbde-47) embryonic exposure modes. Chemosphere. 94, 53-61. 
[29]Mulligan, C.J., D'Errico, N.C., Stees, J., Hughes, D.A.,2012. Methylation changes at NR3C1 in newborns associate with maternal prenatal stress exposure and newborn birth weight. Epigenetics. 7, 853-857.

[30]Martin-Blanco, A., Ferrer, M., Soler, J., Salazar, J., Vega, D., Andion, O., Sanchez-Mora, C., Arranz, M.J., Ribases, M., Feliu-Soler, A., Pérez, V., Pascual, J.C.,2014.Association between methylation of the glucocorticoid receptor gene, childhood maltreatment, and clinical severity in borderline personality disorder. J Psychiatr Res. 57, 34-40.

[31]Kordian, S. , Henning, L. , Ersilia, B. , Siv, K. , Lisa, W. , \& Espen, H. , et al. (2018). Temporomandibular disorders related to stress and hpa-axis regulation. Pain Research and Management, 2018, 1-7.

[33]Marcilla, M., Muñoz, A., Satué, K., 2017. Longitudinal changes in serum catecholamines, dopamine, serotonin, acth and cortisol in pregnant spanish mares. Res Vet Sci. 115, 29-33.

[34]Zhang, W., Li, Q., Deyssenroth, M., Lambertini, L., Finik, J., Ham, J., Huang, Y., Tsuchiya, K.J., Pehme, P., Buthmann, J., Yoshida, S., Chen, J., Nomura, Y.. 2018. Timing of prenatal exposure to trauma and altered placental expressions of hpa-axis genes and genes driving neurodevelopment. J Neuroendocrinol. 30(4), e12581.

[35] Mandic, S., Volkoff, H., 2018. The effects of fasting and appetite regulators on catecholamine and serotonin synthesis pathways in goldfish (carassius auratus). Comp Biochem Phys A. 223, 1-9.

[36]Wu, L.S., Lee, C.S., Weng, T.Y., Wang, K.H., Cheng, A.T. 2016. Association study of gene polymorphisms in gaba, serotonin, dopamine, and alcohol metabolism pathways with alcohol dependence in taiwanese han men. Alcohol Clin Exp Res. 40(2), 284-290.

[37]Xu,Y., Sheng, H., Tang, Z., Lu, J., Ni, X.,2015. Inflammation and increased IDO in hippocampus contribute to depression-like behavior induced by estrogen deficiency. Behav Brain Res. 288,71-78.

\section{Figures}


A

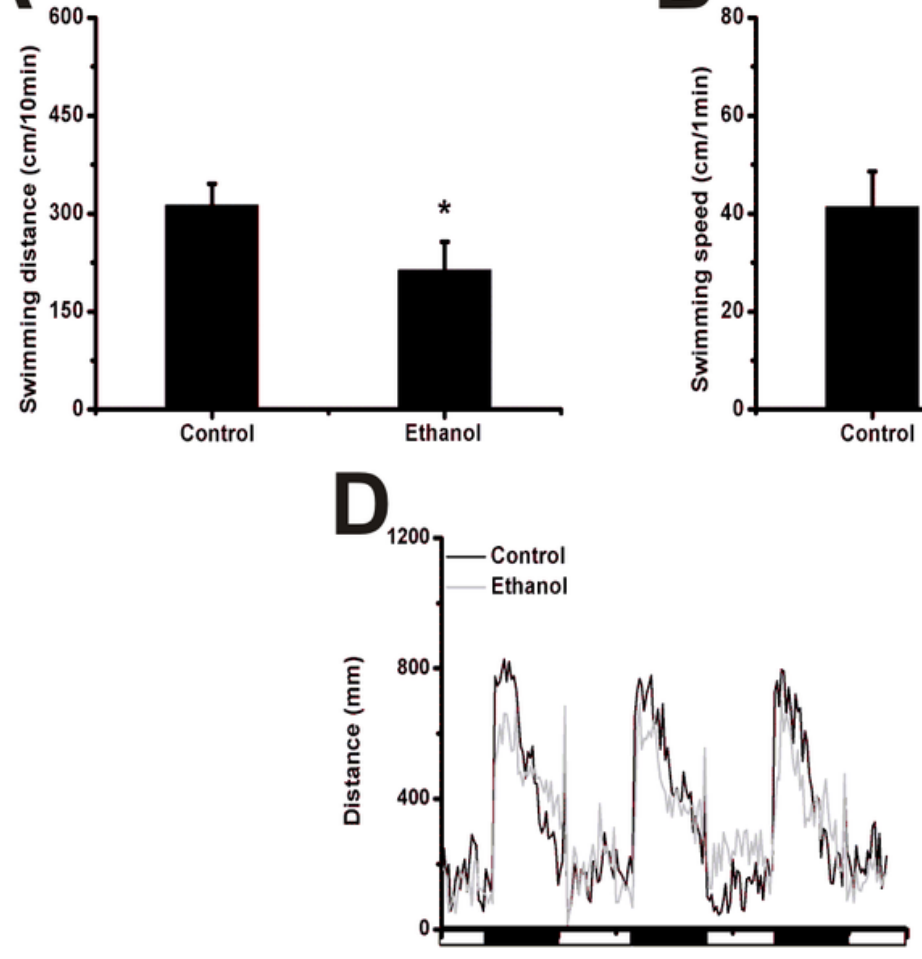

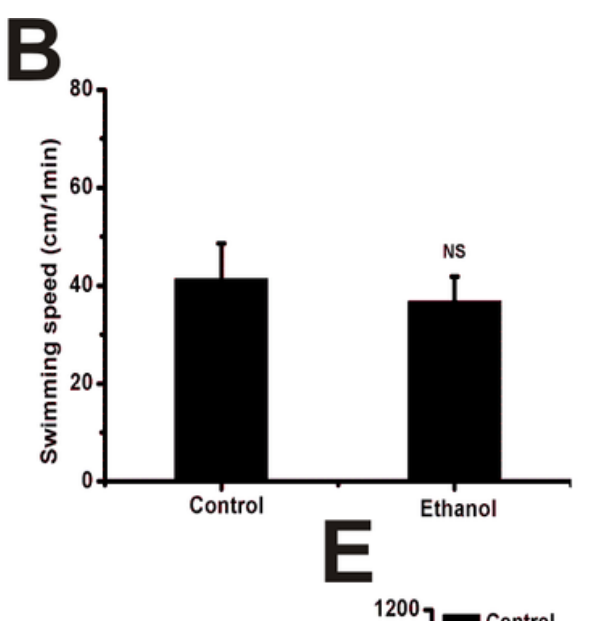
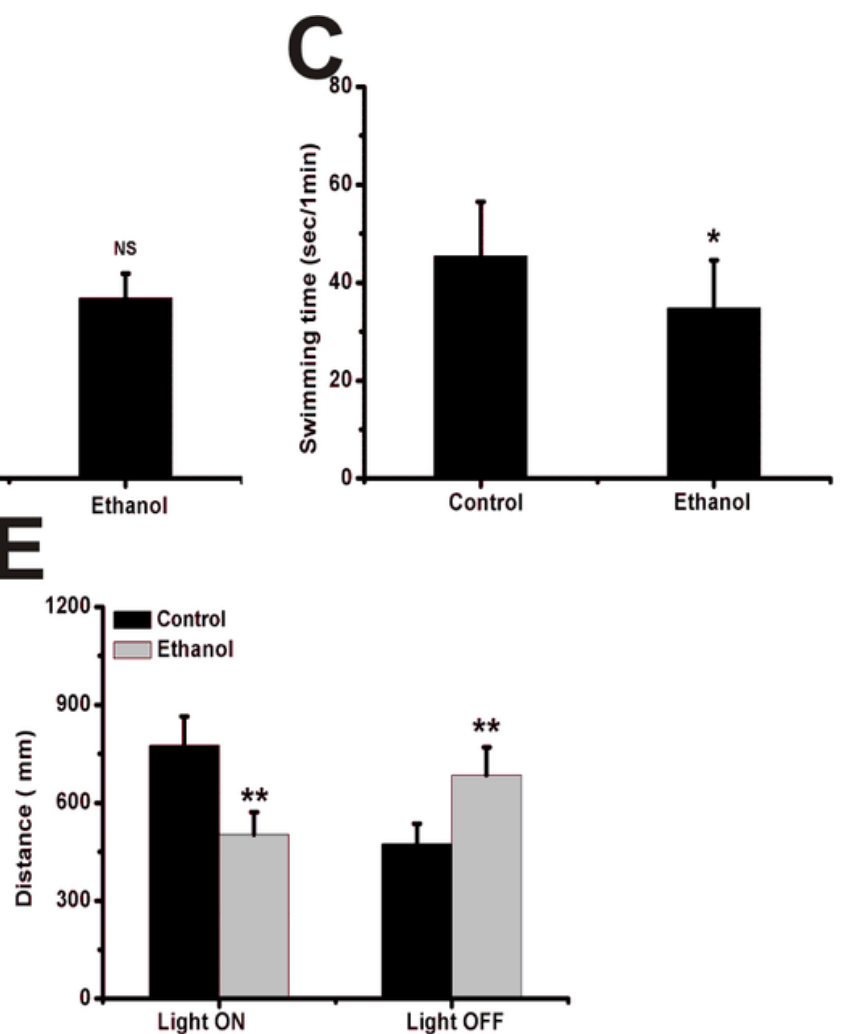

Figure 1

Effects of ethanol exposure on zebrafish locomotor activity. After exposure to alcohol, we investigated $120 \mathrm{hpf}$ zebrafish larvae behaviour. (A) Total swimming distance in 10 min. (B) Swimming speed in 10 min. (C) Swimming time in 10 min. (D) Swimming distance in LD time intervals. (E) Total swimming distance in LD time intervals. Each group, $n=24$. Student's t-test was conducted. ${ }^{*} p<0.05$; ${ }^{\star \star} p<0.01$; $\star \star \star p ~<0.001$.
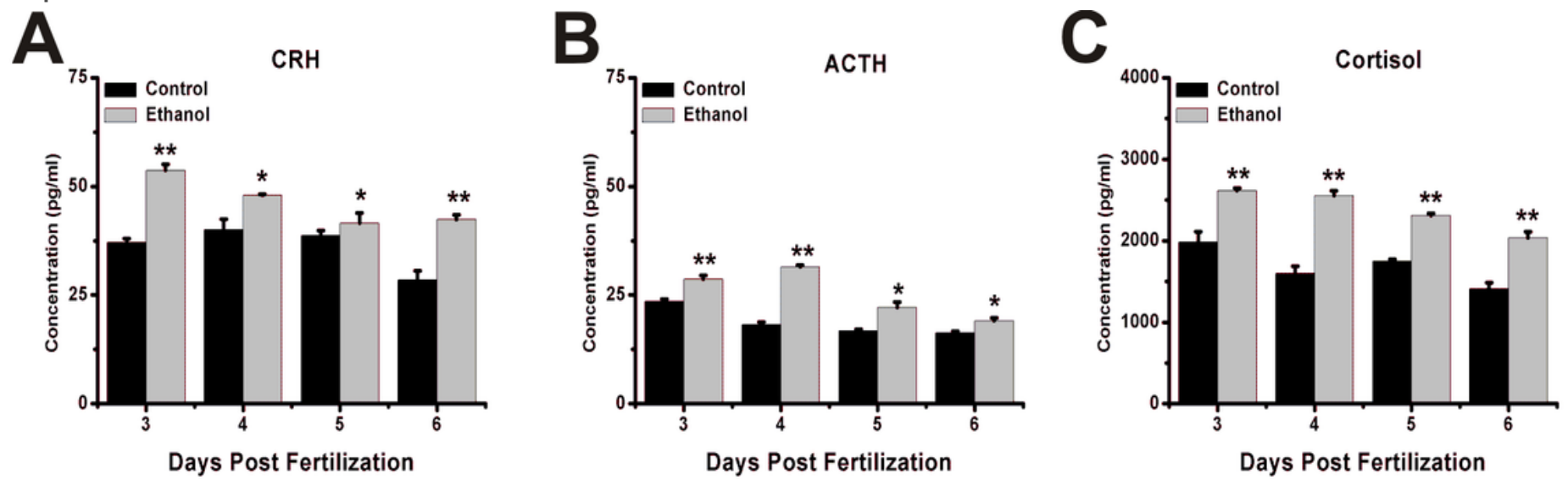

Figure 2

Effects of ethanol on HPA axis hormone secretion in zebrafish larvae. Samples were taken at 3, 4, 5, and $6 \mathrm{dpf}$. (A) CRH levels from 3 to $6 \mathrm{dpf}$. (B) ACTH levels from 3 to $6 \mathrm{dpf}$. (C) Cortisol levels from 3 to $6 \mathrm{dpf}$. 
Three independent experiments were conducted. One-way ANOVAs were performed. ${ }^{\star} \mathrm{p}<0.05 ;{ }^{\star \star} \mathrm{p}<0.01$; $\star \star \star p<0.001$.
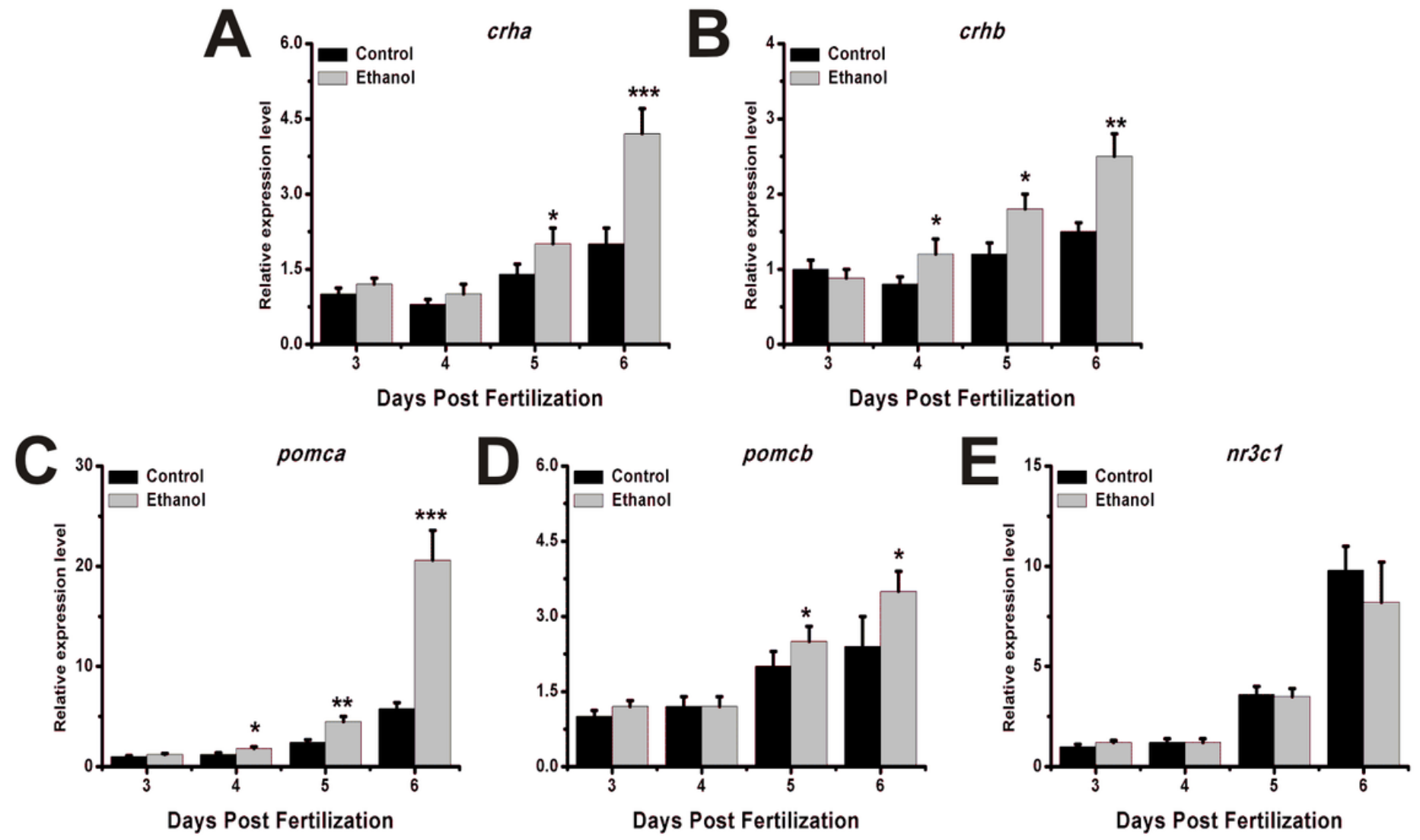

Figure 3

HPA axis gene expression patterns in zebrafish larvae after ethanol exposure. Samples were taken at 3,4 , 5, and $6 \mathrm{dpf}$. The expression patterns of (A) crha, (B) crhb, (C) pomca, (D) pomcb, and (E) gr. Three independent experiments were conducted. One-way ANOVA was performed. ${ }^{\star} p<0.05$; ${ }^{* \star} p<0.01$; $* \star * p<$ 0.001 .

A

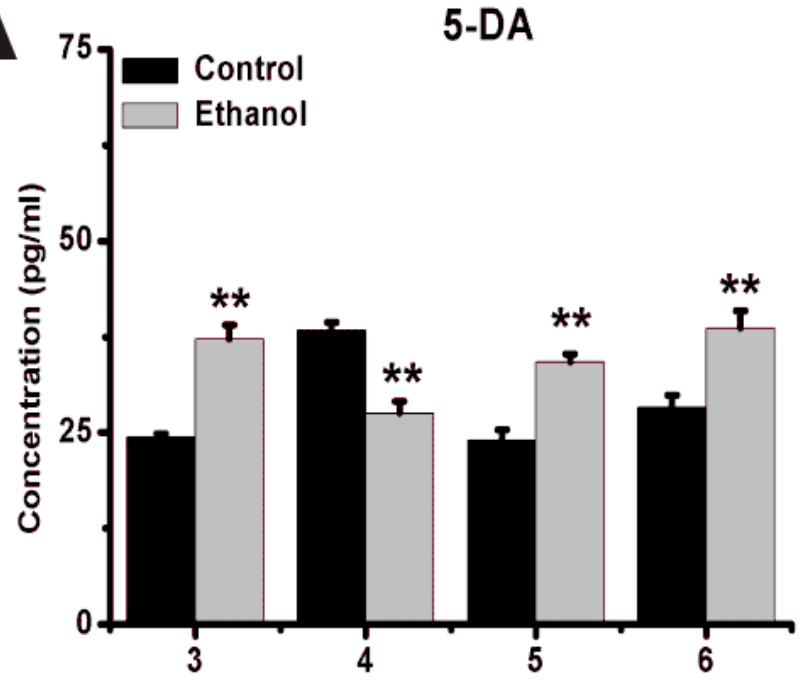

Days Post Fertilization
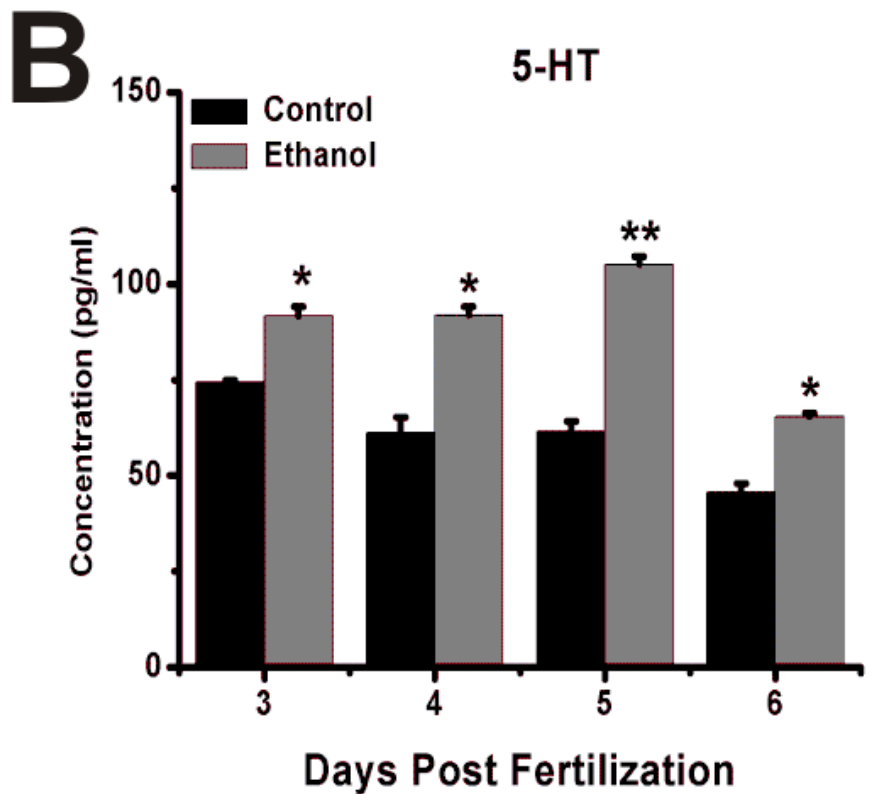
Figure 4

Secretion of neurotransmitters in the zebrafish larvae after ethanol exposure. Samples were taken at 3, 4, 5, and $6 \mathrm{dpf}$. (A) Dopamine concentration from 3 to $6 \mathrm{dpf}$. (B) 5-HT concentration from 3 to $6 \mathrm{dpf}$. Three independent experiments were conducted. One-way ANOVA was performed. ${ }^{\star} p<0.05 ; * \star p<0.01$; ${ }^{\star \star \star} p<$ 0.001 .

A
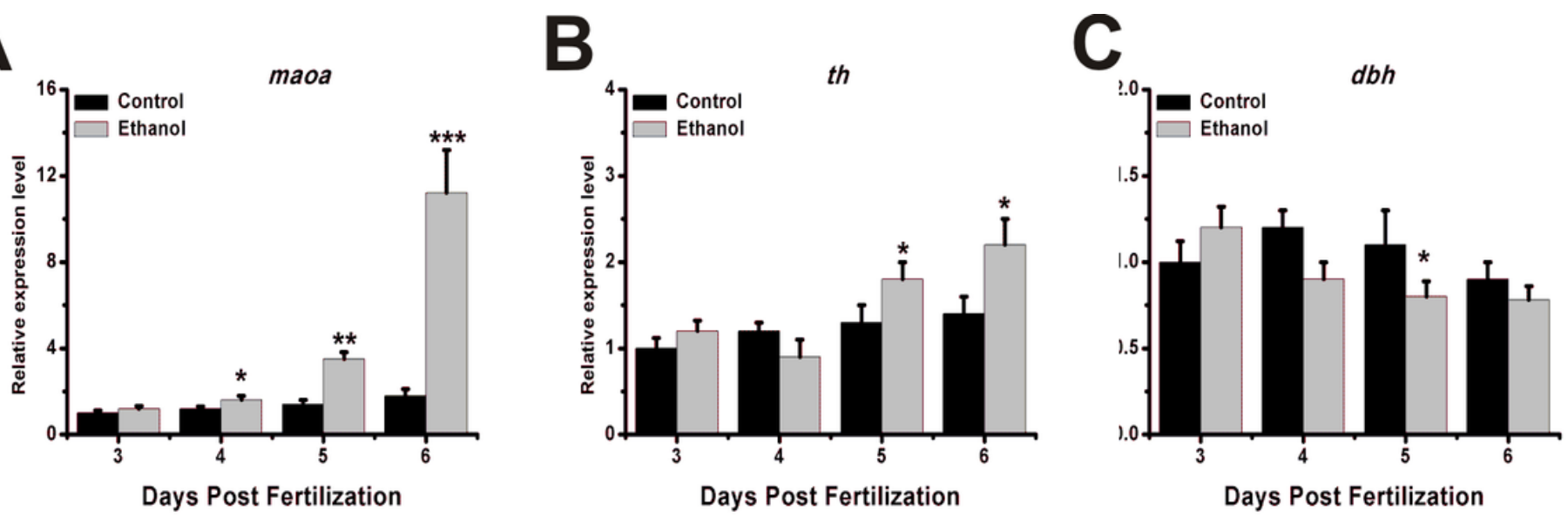

Figure 5

Neurotransmitter metabolism gene expression patterns in zebrafish larvae after ethanol exposure.

Samples were taken at 3, 4, 5, and $6 \mathrm{dpf}$. The expression patterns of (A) maoa, (B) th, and (C) dbh. Three independent experiments were conducted. One-way ANOVA was performed. ${ }^{*} p<0.05 ;{ }^{* \star} p<0.01$; ${ }^{* \star} p<$ 0.001 . 